Results We identified 330 cases of IBD $(49.1 \%$ male, median age $47, \mathrm{IQR}=27$, crude point prevalence rate of 371.5 per $100,000)$. Full diagnostic confirmation was achieved in $100 \%$. The age-standardised point prevalence rate was 359.2 per 100000 . The crude point prevalence rates were 167.8, 158.8 and 45.0 per 100000 for Crohn's disease (CD), ulcerative colitis (UC) and IBD Unspecified (IBDU), respectively. The age-standardised rates were 171.6, 148.1 and 39.5 per 100000 for CD, UC, and IBDU respectively. IBD prevalence steadily increased with age, peaking at 1061 per 100000 in patients older than 85 years. A trend was observed between prevalence and socioeconomic status between suburbs.

Conclusions Sydney exhibited the highest prevalence of IBD in Australasia. The extrapolated estimate for Australia was 89000 people with IBD. Higher socioeconomic status and urbanisation may be contributing factors. The ageing IBD population accounts for the highest prevalence, peaking at greater than 1000 per 100000 . Safer therapies, cancer screening strategies and greater attention towards comorbidities are therefore of increasing importance in managing IBD patients.

\section{IDDF2018-ABS-0038 OUTCOME OF PER ORAL ENDOSCOPIC MYOTOMY IN CHILDREN WITH ACHALASIA WITH A MEDIAN FOLLOW -UP OF 540 DAYS}

Zaheer Nabi*, Radhika Chavan, Mohan Ramchandani, Upender Shava, D Nageshwar Reddy. Asian Institute of Gastroenterology, India

\subsection{6/gutjnl-2018-IDDFabstracts.77}

Background Achalasia cardia (AC) is rare in children, and optimum endoscopic management options are not well known. Per oral endoscopic myotomy (POEM) is a novel treatment modality for AC with excellent results in adult patients. However, the long-term outcomes of POEM are not known in children. In this study, we aim to evaluate the outcome of POEM in AC. Methods We analysed the data of all the children ( $£ 18$ years) with AC who presented to our hospital from September 2013 to January 2018. The outcomes of POEM that were assessed included technical success, clinical success and adverse events. Post-POEM, gastroesophageal reflux (GERD) was assessed with 24 hour $\mathrm{pH}$ impedance, and esophagogastroduodenoscopy (EGD).

Results A total of 43 children (boys-22, girls-21) with mean age, $14.5 \pm 3.41$ years (4-18) with AC underwent POEM during the study period. The subtypes of achalasia according to Chicago classification were - type I- 11, type II- 29, III- 2 and unclassified-1. Eighteen children (41.9\%) had a history of prior treatment including - pneumatic dilatation (15), laparoscopic Heller's myotomy (1) and both dilatation and Heller's myotomy (2). POEM was successfully performed in all the children (technical success-100\%). Intra-operative adverse events occurred in $11(25.6 \%)$ children including retroperitoneal CO2 (7), capnoperitoneum (3) and mucosal injury (1). At a median follow-up of 540 days (66-1594), the clinical success was 95.3\% (39/41). Clinical success was 92.8\% (26/28), 94.4\% (17/18), 92.3\% (12/13), 83.3\% (5/6) at 1, 2, 3, and 4 years follow-up respectively. GERD was assessed in 20 children. Erosive esophagitis was detected in 55\% (11/20). On 24 hour $\mathrm{pH}$ study, high De-Meester score was detected in 53.8\% (7/13) children.

Conclusions POEM is safe and effective for the management of achalasia in children. However, GERD is a potential concern and therefore, randomised comparison with Heller's myotomy combined with fundoplication is warranted in future trials.

\section{IDDF2018-ABS-0040 EFFICACY OF LOW RESIDUE DIET VERSUS CLEAR LIQUID DIET FOR COLONOSCOPY BOWEL PREPARATION IN SPECIAL POPULATIONS (ELDERLY, DIABETICS, CHRONIC KIDNEY DISEASE AND CHRONIC LIVER DISEASE PATIENTS): A SINGLE- BLINDED RANDOMISED NON-INFERIORITY CONTROLLED TRIAL}

Heherson Adriano*. Veterans Memorial Medical Center, Philippines

\subsection{6/gutjnl-2018-IDDFabstracts.78}

Background Ineffective bowel cleansing for colonoscopy results in missed precancerous lesions and increased cost related to early repeat procedures. Better tolerability of bowel preparation may improve patient's compliance and experience and may potentially improve their future participation in colorectal cancer prevention programs without compromising examination quality. The study aims to determine the efficacy and tolerability of low residue diet (LRD) on colonoscopy bowel preparation on special populations compared with the traditional clear liquid diet (CLD).

Methods This study was a prospective single-blinded, randomised controlled non-inferiority trial. A total of 150 patients were included in the analysis. Patients were randomised to receive a low residue diet or a clear liquid diet. The primary outcome is the efficacy or adequacy of bowel preparation and was measured by the Boston Bowel Preparation Score (BBPS). Patient's tolerability, hunger score and overall satisfaction were assessed as secondary outcomes.

Results Low residue diet (LRD) bowel preparation was noninferior to Clear liquid diet (CLD) bowel preparation (LRD 7.00 vs CLD $6.9 \mathrm{p}=0.7724)$. Overall, patient's tolerability and satisfaction were better in the LRD group than the CLD group. The present study showed that the proportion of patients having abdominal pain/cramping/discomfort and bloatedness were significantly lower in the LRD group (16\% and 26.7\%) compared to CLD group (34.7\% and 44\%).

Conclusions Patients on an LRD for bowel preparation achieved an adequate bowel preparation that is non-inferior to patients on a CLD. Furthermore, the study showed that patients' tolerability and overall satisfaction were better on the LRD and more willing to repeat the same bowel regimen as compared to CLD.

\section{IDDF2018-ABS-0043 ILEOCAECAL CROHN'S DISEASE MISDIAGNOSED AS TUBERCULOSIS IN YOUNG LADY- A CASE REPORT}

Megha Shah*. Community Health Center, Mehmedabad, Gujarat, India

\subsection{6/gutjnl-2018-IDDFabstracts.79}

Background Large number of patients with Crohn's disease (CD) are initially misclassified as intestinal tuberculosis (TB) in TB endemic areas, and some patients develop activation of latent $\mathrm{TB}$ during treatment for $\mathrm{CD}$ with biologics and immunosuppressants, we present a case of young lady who was 